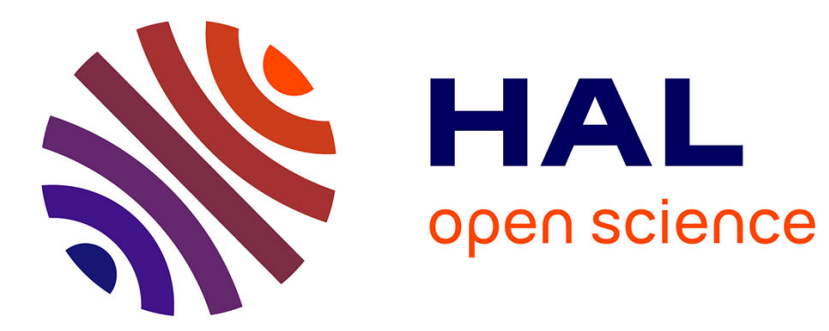

\title{
Large fault-tolerant interconnection networks
}

Jean-Claude Bermond, Nathalie Homobono, Claudine Peyrat

\section{To cite this version:}

Jean-Claude Bermond, Nathalie Homobono, Claudine Peyrat. Large fault-tolerant interconnection networks. Graphs and Combinatorics, 1989, 5 (1), pp.107-123. 10.1007/BF01788663 . hal-02508728

\section{HAL Id: hal-02508728 \\ https://hal.inria.fr/hal-02508728}

Submitted on 15 Mar 2020

HAL is a multi-disciplinary open access archive for the deposit and dissemination of scientific research documents, whether they are published or not. The documents may come from teaching and research institutions in France or abroad, or from public or private research centers.
L'archive ouverte pluridisciplinaire HAL, est destinée au dépôt et à la diffusion de documents scientifiques de niveau recherche, publiés ou non, émanant des établissements d'enseignement et de recherche français ou étrangers, des laboratoires publics ou privés. 


\title{
Large Fault-Tolerant Interconnection Networks
}

\author{
J.-C. Bermond, N. Homobono and C. Peyrat \\ Laboratoire de recherche en Informatique, Universite Paris-Sud, Bat 490, 91405 Orsay, France
}

\begin{abstract}
This paper deals with reliability and fault-tolerant properties of networks. We first survey general reliability properties of networks, in particular those concerning diameter vulnerability. Then we study in details reliability properties of some families of networks in particular de Bruijn and Kautz networks and their generalizations which appear as very good fault-tolerant networks.
\end{abstract}

\section{Introduction}

An important consideration in the design of communication networks as well as distributed computer systems is the interconnection network. This network is usually modeled by a graph or a digraph in which the vertices represent the switching elements or processors. Communication links are represented by edges if they are bidirectional or arcs if they are unidirectional. Several factors have to be taken into account in the design of interconnection networks (see for instance the book by W.D. Hillis [59] or [4]):

- communication delays between processors must be short: the graph must have a small diameter or mean distance.

- the number of processors directly connected to a given processor is limited: the graph has a given maximum degree.

- the routing of the messages must be simple and distributed if possible.

- finally an interconnection network must be fault-tolerant. Indeed in a system consisting of a large number of processors, the probability that a processor or a link happens to be faulty becomes important.

One minimal requirement is that the system still works in case of node or link failures. This means that the associated graph is sufficiently connected. Furthermore one can also demand that the message delay does not increase too much. This means that the graph obtained after deletion of vertices or edges still has a small diameter, good routings and so on. All this leads one to consider different kinds of reliability or fault-tolerance properties and to define different measures of vulnerability.

Here we focus on relability results. We restrict ourselves to mensures involving diameier properties. For results involving connectivity only, see the survey of W. Mader [78]. We also consider only deterministic problems. For results involving probabilities of failures see the survey of F.T. Boesch [14] or the book of C.J. 
Colbourn [33]. Essentially we distinguish three kinds of results:

a) general reliability properties of networks,

b) reliability properties of some particular networks or families of networks,

c) construction of networks having given reliability properties.

We will quickly survey some recent results in part a, which is well developed.

Part $b$ has also received some attention. In particular, we summarize the vulnerability properties of de Bruijn and Kautz networks, which have been investigated recently. We add some new results on this topic. Part $\mathrm{c}$ is somewhat less developed. We will indicate further possible directions of research. Other results can be found in Pradhan's book [91].

\section{Definitions and Notation}

We will represent the nodes of an interconnection network by the vertices and the links by the edges (or arcs) of an undirected (or directed) graph $G=(V, E)$. All the definitions not given here can be found in [5]. We precise some notation in the undirected case. Similar notation will be used for digraphs.

Let $\Gamma(x)$ denote the set of neighbors of a vertex $x$. The degree $d(x)$ of $x$ is the cardinality of $\Gamma(x)$. The maximum degree $\Delta$ of $G$ is the maximum over all the degrees of the vertices of $G$. The minimum degree $\delta$ of $G$ is the minimum over all the degrees of the vertices of $G$. The distance $d(x, y)$ between $x$ and $y$ is the length of a shortest path between $x$ and $y$. The diameter $D(G)$ of $G$ is the maximum distance over all the pairs of vertices. The mean distance of $G$ is the average of the distances over all the pairs of vertices in the graph. We call $(\Delta, D)$-graph a graph with maximum degree $\triangle$ and diameter $D$. The number of vertices of a $(\Delta, D)$-graph is bounded by the Moore

$$
n(\Delta, D)=1+\Delta \frac{(\Delta-1)^{D}-1}{\Delta-2} \quad \text { if } \Delta>2 .
$$

The $(\Delta, D)$-problem, which consists in finding a $(\Delta, D)$-graph with the maximum number of vertices, has been extensively studied. See [10] for further details.

A graph $G$ is $k$-connected if there exist $k$ internally vertex disjoint paths between any pair of vertices. The connectivity $\kappa(G)$ of $G$ is the greatest integer $k$ such that $G$ is $k$-connected. Similarly a graph is $l$-connected if there exist $l$ edge-disjoint paths between any pair of vertices. The edge-connectivity $\lambda(G)$ of $G$ is the greatest integer $l$ such that $G$ is $l$-edge-connected.

A routing $\rho$ is a function in which $\rho(x, y)$ is a path in $G$ from $x$ to $y$. A minimal length routing is a routing in which all the paths are shortest paths.

Let $F$ be a set of vertices and/or edges, such that $|F|<\kappa(G)(F$ corresponds to the set of faults in the network) and let $\rho$ be a routing. Following D. Dolev, J. Halpern, B. Simons and R. Strong [34], the surviving route graph $R(G, \rho) / F$ is a directed graph with vertex set $V-V(F)$. There is an arc from $x$ to $y$ in $R(G, \rho) / F$ if $\rho(x, y)$ contains neither vertices nor edges of $F$. In this case, $\rho(x, y)$ is said to avoid $F$. 
F.R.K. Chung, E.G. Coffman, M.I. Reiman and B.E. Simon ([31]) defined what they call the forwarding index. The forwarding index $\xi(G, \rho)$ of a network $G$ with a given routing $\rho$ is the maximum number of paths of the routing passing through a given vertex. It corresponds to the maximum amount of forwarding that any node has to do in a communication network with a fixed routing. M-C. Heydemann, J-C Meyer and D. Sotteau [58] defined the analogous concept $\Pi(G, \rho)$ for edges instead of vertices. The edge forwarding index corresponds to the maximum load of the network.

The vertex-forwarding (resp. edge-forwarding) index of a graph $\xi(G)(\operatorname{resp} . \Pi(G))$ is the minimum of $\xi(G, \rho)$ (resp. $\Pi(G, \rho))$ taken over all possible routings $\rho$.

\section{Some General Reliability Results}

There are a lot of results concerning the connectivity and the edge-connectivity of graphs or digraphs. See for example W. Mader's survey [78]. For generalizations of the classical notion of connectivity see the survey by F.T. Boesch [14] or the recent article by C. Dwork, D. Peleg, N. Pippenger and E. Upfal [39].

As we said in the introduction, the results involving both connectivity and diameter (or path length) as vulnerability measure are very interesting for the construction of fault-tolerant networks. This gives rise to many interesting extremal diameter vulnerability problems. Many results can be found in one of the earlier surveys [7, 8, 28, 29].

We present below some recent results.

A natural problem considered only recently by F.R.K. Chung and M.R. Garey [32] consists in studying how much the diameter can increase after the deletion of vertices or edges.

They denoted by $f(t, D)$ the maximum possible diameter for a graph obtained from a $(t+1)$-edge-connected graph of diameter $D$ by deleting $t$ edges. They showed that:

$$
(t+1)(D-2) \leq f(t, D) \leq(t+1) D+t \quad \text { if } D \geq 4 .
$$

Recently A. Schoone, H. Bodlaender and J. van Leeuwen [100] found a shorter proof of this result and showed that the associated problems are NP-complete. Furthermore they improved the bounds. They also showed that:

$$
f(2, D)=3 D-1 \text { and that } f(3, D)=4 D-2 .
$$

The fact that $f(1, D)=2 D$ was established earlier by J. Plesnik [87]. R. Kerjouan [72] independently established the values of $f(2, D)$ and $f(3, D)$ and obtained the best of the known bounds:

$$
\begin{gathered}
\text { if } D \geq 4 \text { is even, }(t+1) D-t \leq f(t, D) \leq(t+1) D-t+3 . \\
\text { if } D \geq 5 \text { is odd, }(t+1)(D-2)+6 \leq f(t, D) \leq(t+1) D-t+3 .
\end{gathered}
$$

C. Peyrat [86] proved that $f(t, 2)=4$ and that $3 \sqrt{2 t}-3 \leq f(t, 3) \leq 3 \sqrt{2 t}+4$.

The analogous problem for the mean distance has been studied by O. Favaron, M. Kouider and M. Maheo [48]. The situation is completely different, in the sense 
that there exist networks, with a small mean distance, such that, if some link fails, the mean distance becomes very large. More precisely, they have shown that after the deletion of one edge the ratio mean distance of the original graph over mean distance of the resulting graph can be greater than $\frac{\sqrt{n}}{4}$.

In case of deletion of vertices, F.R.K. Chung and M.R. Garey [32] proved that the diameter of the resulting graph is not bounded. However J. Bond and C. Peyrat [19] have shown that the diameter of the resulting graph remains small if the degree is bounded or the number of vertices is large enough. For example, they showed that if $G$ is 2-connected, has maximum degree $\Delta$ and if $D^{\prime}$ denotes the diameter of the graph obtained from $G$ after the deletion of $s$ vertices, then

$$
\begin{gathered}
D^{\prime} \leq s \Delta(D-1)-1 \quad \text { if } D \text { is even and } \Delta \geq 5, \\
D^{\prime} \leq s \Delta(D-2)+6 \text { if } D \text { is odd. }
\end{gathered}
$$

One of the oldest problem in this area was posed by K. Vijayan and U.S.R. Murty [108]. Given $n, D, D^{\prime}$ and $t$, find the minimum number of edges in a graph on $n$ vertices, of diameter $D$, with the property that after the removal of $t$ edges, the resulting graph has diameter no more than $D^{\prime}$. In view of the above result, when $D^{\prime}$ is large (greater than $(t+1) D-t+3$ ), the problem is reduced to finding the minimum number of edges of a graph on $n$ vertices, with diameter $D$ and edgeconnectivity $t+1$. This case has been solved by B. Bollobas [17]. But the general problem is far from being solved. See one of the surveys [7, 29]. An interesting particular case is when $D^{\prime}=D$. In that case, the problem is to determined $g(n, D, t)$, the minimum number of edges of a graph on $n$ vertices and diameter $D$, such that after deleting $t$ edges, the diameter of the resulting graph is at most $D$. The case $t=1$ has been solved only recently by F.R.K. Chung [30] who proved that $g(n, D, 1)=n-1+\left[(n-1-\varepsilon) /\left[\frac{2 D}{3}\right]\right\rceil$, where $\varepsilon=1$ if $D \equiv 1(\bmod 3)$ and $\varepsilon=0$ otherwise. In [30], F.R.K. Chung gave also upper bound for $g(n, D, t)$ for $t \geq 2$.

Similar problems can be asked (see [29]) with constraints on the maximum degree $\Delta$.

Instead of considering diameter of the graph obtained after deletion of vertices or edges, one can ask for the existence of $p$ vertex (or edge) disjoint paths of length at most $k$ between any pair of vertices. This notion arises in many communication problems and in distributed computing when a message can not use some faulty or over-loaded routes or is to be sent by different routes (for example to reach a byzantine agreement).

Let us denote by $I_{k}(x, y)$ the maximum number of vertex disjoint paths of length at most $k$ between the two vertices $x$ and $y$ and by $S_{k}(x, y)$ the minimum number of vertices whose deletion yields a graph in which there is no path of length at most $k$ between the vertices $x$ and $y$. Clearly $I_{k}(x, y) \leq S_{k}(x, y)$.

If there are no restriction on $k$ (or equivalently if $k=n-1$ ), then by Menger's theorem the maximum number of vertex disjoint paths between $x$ and $y$ is equal to the minimum number of vertices whose removal destroy all the paths between $x$ and $y$. That is $I_{n-1}(x, y)=S_{n-1}(x, y)$. R.C. Entringer, D.E. Jackson and P.J. Slater 
[40] and L. Lovasz, V. Neuman-Lara and M. Plummer [75] have proved that $I_{k}(x, y)=S_{k}(x, y)$ for $k=2,3,4$ or $k=d(x, y)$. However, in the general case, there is no equality (in fact $\frac{S_{k}(x, y)}{I_{k}(x, y)}$ can be as large as $\left.\left\lfloor\frac{k+1}{3}\right\rfloor\right)$.

A. Itai, Y. Perl and Y. Shiloah [69] proved that the computing of $I_{k}(x, y)$ is NP-complete when $k \geq 5$. Recently D. Ronen and Y. Perl [97] have given an heuristic which gives optimal solutions for $k \leq 4$ and experimentally very good ones otherwise. Note that for the related problem of finding a maximum number of vertex disjoint paths of minimum total length, a polynomial algorithm is given by J.W. Surballe [107].

Finally, let us note that no global mengerian theorem holds, as shown by S.M. Boyles and G. Exoo [22], G. Exoo [44] and J.A. Bondy and P. Hell [21]. Let the "persistence" $\rho_{0}(G)$ of a graph $G$ be the minimum number of vertices which must be removed in order to increase the diameter (or yield a trivial graph). The preceding authors constructed examples of graphs such that $\rho_{0}(G)$ is strictly larger than $\min I_{D}(x, y)$ taken over all pairs of vertices. Similar results have been obtained for edge disjoint paths (see also J. Hartman and I. Rubin [56]).

So, it appears interesting to find conditions under which a general graph has $p$ disjoint paths of length at most $k$. Results involving various combinations of the connectivity, the minimum degree, the edge-connectivity and the number of vertices have been recently given by R.J. Faudree and al [47].

An other problem is to find conditions involving the diameter and insuring that the connectivity $\kappa$ (or edge connectivity $\lambda$ ) of a graph is maximum that is equal to its minimum degree (recall that for any graph $\kappa(G) \leq \lambda(G) \leq \delta(G)$ ).

Perhaps, the oldest result is due to J-L. Jolivet [70] and says that if a digraph has diameter 2 then $\lambda=\delta$. The corollary for undirected graph has also been obtained by J. Plesnik [88]. More recently, M. Imase T. Soneoka and K. Okada [67] studied the connectivity of large digraphs with given degree and diameter, proving that the connectivity of a digraph with $n$ vertices and minimum degree $d$ is at least $\frac{n(d-1)}{d^{D}+d^{2}-d-1}$.

The undirected case was considered by A.H. Esfahanian [42] and by M. Imase, T. Soneoka and H. Nakada [104]. T. Soneoka, H. Nakada, M. Imase and C. Peyrat [106] showed that if $\Delta \geq 3$, the graph is maximally connected in the sense that the connectivity $\kappa=\delta$ when $n>(\delta-1)(\Delta-1)^{D-1}+2$ and that this bound is best possible. Indeed there exist graphs with $(\delta-1)(\Delta-1)^{D-1}+2$ vertices which are not 2-connected. Similar results were obtained for the edge-connectivity.

In $[104,106]$ it is also proved that if $g$ is the girth of the graph, then

$$
\begin{gathered}
\kappa=\delta\left\{\begin{array}{lll}
\text { if } & D \leq g-2 & g \text { odd } \\
\text { if } & D \leq g-3 & g \text { even }
\end{array}\right. \\
\lambda=\delta\left\{\begin{array}{lll}
\text { if } & D \leq g-1 & g \text { odd } \\
\text { if } & D \leq g-2 & g \text { even }
\end{array} .\right.
\end{gathered}
$$

These bounds are best possible. The result that if $D=2$, then $\lambda=\delta$ follows as a 
corollary. Furthermore these results show that some of the best of the known $(\triangle, D)$-graphs have maximum connectivity.

In the directed case, similar results have been obtained by J. Fabrega, M.A. Fiol and M. Escudero [45]. For any directed loopless graph $G$, they define $l(G)$ as the greatest integer $l$ such that, for any two vertices $x$ and $y$ in $G$, the following property holds: a) if $d(x, y)<l$, then there exists a unique shortest path from $x$ to $y$ and no path of length $d(x, y)+1$ from $x$ to $y$; b) if $d(x, y)=l$, then there exists a unique shortest path from $x$ to $y$.

They proved the following result

$$
\begin{array}{ll}
\lambda=\delta & \text { if } D<2 l(G) \\
\kappa=\delta & \text { if } D \leq 2 l(G)-1
\end{array}
$$

This result has many interesting corollaries. It contains the preceding results concerning the girth. Indeed, in the undirected case, the parameter $l=\left\lfloor\frac{g-1}{2}\right\rfloor$. An other corollary is that if $L^{k}(G)$ is the $k$-iterated line digraph of $G$, then if $k \geq$ $D-2 l(G)$ (resp. $k \geq D-2 l(G)+1$ ), then the edge-connectivity (resp. the connectivity) of $G$ is equal to its minimum degree.

Finally concerning the surviving route graph, interesting results and problems can be found in the articles of A. Broder, D. Dolev, M. Fisher, J. Halpern, B. Simons and R. Strong ([23] and [34]). They proved various results on general graphs and give open problems. Some of them have been solved by P. Feldman in [49]. He proved for example that if $\rho$ is a minimal length routing and $F$ is a set of edges, then the diameter of the surviving route graph satisfies $D(R(G, \rho) / F) \leq 2|F|+1$.

In [85] D. Peleg and B. Simons constructed fault tolerant routings for several families of graphs (in particular graphs with $\Delta \leq c n^{1 / 3}$ ). With these routings, the surviving route graphs have diameter bounded by 4 or 6 . In [66] M. Imase and Y. Manabe proved that if a graph has connectivity $\kappa$ and more that $2 \kappa^{2}$ vertices, then it has a routing for which the diameter of the associated surviving route graph is at most 3. In [79] Y. Manabe, M. Imase and T. Soneoka gave sufficient conditions for $k$ connected digraphs $(k \neq 2,4)$ to have a routing $\rho$ such that $D(R(G, \rho) / F) \leq 6$ for $|F|<k$.

General bounds concerning the forwarding indices can be found in the article of F.R.K. Chung, E.G. Coffman, M.I. Reiman and B. Simon [31] and M-C. Heydemann, J-C Meyer and D. Sotteau [58]. In these articles, values of indices for particular graphs including the D-cube are given. Recently M-C Heydemann, J-C Meyer, J. Opatrny and D. Sotteau [57] proved that the problem of finding a routing minimizing $\xi(G)$ (or $\Pi(G))$ for a given graph $G$ is NP-complete.

\section{Reliability Results for Particular Families of Graphs}

Some of the results obtained for general graphs can be used to find the connectivity or the "diameter vulnerability" of certain networks, in particular for large known $(\triangle, D)$-graphs. That is the case for some of these graphs by the results of [106]. But 
generally one must use the specific properties of the considered network to obtain more precise results and also to obtain the routings in case of node or link failures.

Among the most popular interconnection networks are now the hypercubes or $D$-cubes. Recall that the $D$-cube $C_{D}$ is the graph whose vertices are the $D$-tuples on the alphabet $\{0,1\}$; two vertices being adjacent if they differ in exactly one coordinate. This graph is $D$-regular, has diameter $D$ and $2^{D}$ vertices. The $D$-cubes have very good fault-tolerant properties due to the fact that there are $D$ disjoint paths of length at most $D+1$ between any pair of vertices. There exist also fault-tolerant routings for these graphs. For example, A. Broder, B. Dolev, M. Fischer, B. Simons [23] showed that the diameter of the surviving route graph of the $D$-cube $C_{D}$ satisfies

$$
D\left(R\left(C_{D}, \rho\right) / F\right) \leq 3
$$

if $\rho$ is any minimal length routing and $|F|<D$. They also showed that there exists a particular minimal length routing $\lambda$ such that $D\left(R\left(C_{D}, \lambda\right) / F\right)=2$. The $D$-cubes have also good forwarding indices (see $[31,58]$.)

Some of these properties follow from the fact that the cubes have nice symmetry properties, and are in fact Cayley graphs. Recall that the Cayley digraph associated to a group $\Gamma$ and a set of generators $S$ has the elements of the group $\Gamma$ as vertex set. There is an arc from the vertex $g$ to the vertex $h$ if $g^{-1} h$ belongs to $S$.

A Cayley graph corresponds to the symmetric case $S=S^{-1}$. If the group is the group of the integers modulo $n$ and $S=\left\{s_{1}, s_{2}, \ldots, s_{k},-s_{1},-s_{2}, \ldots,-s_{k}\right\}$, we obtain the so-called circulant graph $G\left(n ; \pm s_{1}, \pm s_{2}, \ldots, \pm s_{k}\right)$, where the vertices are the integer modulo $n$ and vertex $i$ is joined to the $2 k$ vertices $i \pm s_{1}, i \pm s_{2}, \ldots, i \pm s_{k}$.

The study of the diameter of circulant graphs or digraphs has been done by different authors. See for example the articles by C.K. Wong and D. Coppersmith [111], F.T. Boesch and J. Wang [16], M.A. Fiol, J.L.A. Yebra, I. Alegre and M. Valero [51] or J-C. Bermond, G. Illiades and C. Peyrat [12] and the survey of J-C. Bermond, F. Comellas and F. Hsu [9]. Diameters of other Cayley graphs, like those of cube connected cycles (defined by F. Preparata and J. Vuillemin [93]) or star graphs (defined by S.B. Akers and D. Harel and B. Krishnamurthy [1]) have been determined. Furthermore, Cayley graphs are, in many cases, the largest of the known $(\Delta, D)$ graphs (see the articles of J. Bond, C. Delorme and W.F. de la Vega [18], L. Campbell et al. [25], G.E. Carlsson, J.E. Cruthirds, H.B. Sexton and C.G. Wright [26], D.V. Chudnovsky, G.V. Chudnovsky and M.M. Denneau [27]).

The connectivity of circulant or Cayley graphs has been well studied too (see the survey of F.T. Boesch and R. Tindell [5] or the article by S.B. Akers and B. Krishnamurthy [2], E. Van Doorn [35] for circulant digraphs and the article by Y.O. Hamidoune [55] for Cayley graphs). Y.O. Hamidoune developed general tools using atom's theory. In particular he proved that if $\Gamma$ is a finite group, $S$ is a minimal generating set of $\Gamma$, and $S^{\prime}$ a subset of $S^{-1}$, then the connectivity of the Cayley graph associated to $\Gamma$ and $S \cup S^{\prime}$ is equal to $\left|S \cup S^{\prime}\right|$.

There exist also different results on the connectivity of vertex or edge transitive graphs (see the survey by W. Mader [78]). Some useful results are the following: If $G$ is an edge transitive graph then $\kappa=\lambda=\delta$ (W. Mader [76] and M. Watkins [110]). If $G$ is a vertex transitive graph then $\lambda=\delta$ and if furthermore $G$ has no $K_{4}$, 
then $\kappa=\delta$ (W. Mader $[76,77])$. For example, the last results can be used to show that a general family of large $(\Delta, D)$-graphs is maximally connected (see the article of D. Amar [3] who proved it directly by exhibiting the disjoint routings).

Recent results have been obtained concerning the diameter vulnerability of circulant graphs. Partial results concerning double loop graphs can be found in [12] and for loop digraphs in articles by M.A. Fiol, L.A. Yebra, I. Alegre and M. Valero [51] and P. Morillo, F. Comellas, M.A. Fiol [81].

M. Escudero, J. Fabrega and P. Morillo [41] have proved that in any doubleloop graph, there exist a shortest path routing for which the associated surviving route graph has diameter 2 in case of up to 3 failures.

J. Bond and C. Peyrat [19] gave some results concerning the diameter vulnerability of particular products of graphs used to construct good $(\Delta, D)$-networks. M.S. Krishnamurthy and B. Krishnamoorthy [73] gave results on the existence of $\kappa$ disjoint paths of length at most $D^{\prime}$ in the cartesian sum of graphs (and not on diameter vulnerability as stated in their theorem). They also gave results on the existence of $\kappa$ vertex disjoint paths in cube connected cycles.

Maybe the most interesting families of networks are the de Bruijn and Kautz graphs and their generalizations, as they are the best of the $(\Delta, D)$-graphs known at the present time for large values of $\Delta$. These graphs are not vertex transitive, but they have a lot of nice properties comparable to those of the $D$-cubes. In the next paragraph, we collect results concerning their reliability properties.

\section{Reliability Properties of de Bruijn and Kautz Networks}

Definitions. First we give three equivalent definitions of the de Bruijn and Kautz digraphs.

\section{From an alphabet.}

The de Bruijn digraph $B(d, D)$ (defined in [24]) with in- and out-degree $d$ and diameter $D$ is the digraph whose vertices are labeled with words of length $D$ from an alphabet of $d$ letters. There is an arc from a vertex $x$ to a vertex $y$ if and only if the last $D-1$ letters of $x$ are the same as the first $D-1$ letters of $y$. In other words there is an arc from $\left(x_{1}, x_{2}, \ldots, x_{D}\right)$ to the vertices $\left(x_{2}, \ldots, x_{D}, \alpha\right)$, where $\alpha$ is any letter. This digraph has $d^{D}$ vertices.

The Kautz digraph $K(d, D)$ (defined in [71]) with in- and out-degree $d$ and diameter $D$ is the digraph obtained from the de Bruijn digraph $B(d+1, D)$ by removing the words in which there are two consecutive identical letters. That is, the vertices are labeled with words $\left(x_{1}, \ldots, x_{D}\right)$, where $x_{i}$ belongs to an alphabet of $d+1$ letters, and $x_{i} \neq x_{i+1}$, for $1 \leq i \leq D-1$. The vertex $\left(x_{1}, \ldots, x_{D}\right)$ is joined to the $d$ vertices $\left(x_{2}, \ldots, x_{D}, \alpha\right)$, where $\alpha$ is any letter different from $x_{D}$. This digraph has $d^{D}+d^{D-1}$ vertices.

From line digraphs iterations.

A definition using line digraphs iterations has been given by M.A. Fiol, J.L.A. Yebra and I. Alegre [52]. Recall that the line digraph of a digraph $G$ is the digraph $L(G)$ 
whose vertices represent the arcs of $G$. There is an arc in $L(G)$ from $x$ to $y$ if and only if $x$ represents the arc $(u, v)$ in $G$ and $y$ represents the arc $(v, w)$ in $G$ for some vertices $u, v$ and $w$ in $G$. If $G$ is $d$-regular and has diameter $D$, then $L(G)$ is $d$-regular and has diameter $D+1$ (except when $G$ is a circuit).

Let $K_{d}^{\circ}$ be the complete digraph on $d$ vertices with a loop on each vertex, then $L^{D-1}\left(K_{d}^{\circ}\right)$ is the de Bruijn digraph $B(d, D)$. Now let $K_{d+1}$ be the complete digraph on $d+1$ vertices (with no loops), then $L^{D-1}\left(K_{d+1}\right)$ is the Kautz digraph $K(d, D)$.

\section{Generalization.}

The third definition is arithmetic and gives rise to a generalization of these digraphs for every integer $n$. The generalization of the de Bruijn digraphs was considered by M. Imase and M. Itoh [64] and by S.M. Reddy, D.K. Pradhan and J.G. Kuhl [96]. The $R P K_{d, n}$ digraph has the set of integers modulo $n$ as vertex set. Its arc set $A$ is defined as follows: $A=\{(x, y) / y \equiv d x+a, 0 \leq a \leq d-1\}$.

The generalization of the Kautz digraphs was first studied by M. Imase and M. Itoh [65]. The $\Pi_{d, n}$ digraph has the set of integers modulo $n$ as vertex set. Its arc set $A$ is defined as follows: $A=\{(x, y) / y \equiv-d x-a, 1 \leq a \leq d\}$.

For particular values of $n$, we find again the de Bruijn and Kautz digraphs:

If $n=d^{D}$, then $R P K_{d, n}=B(d, D)$.

If $n=d^{D}+d^{D-1}$, then $\Pi_{d, n}=K(d, D)$.

$R P K_{d, n}$ has $n$ vertices and is $d$-regular (provided that $n>d$ ). Its diameter is $\left\lceil\log _{d} n\right\rceil$. See [96] for a proof.

$\Pi_{d, n}$ has $n$ vertices and is $d$-regular (provided that $n>d$ ). Its diameter is at most $\left\lceil\log _{d} n\right\rceil$. If $n$ is $d^{p}+d^{p-q}$, (where $p$ and $q$ are two integers, with $q$ odd and $q \leq p$ ), then its diameter is $\left\lceil\log _{d} n\right\rceil-1$. See [65] for a proof.

\section{Undirected case.}

De Bruijn and Kautz undirected graphs are obtained from the associated digraphs by removing the orientation, the loops and the parallel edges if any. In the following we will denote by $U G$ the undirected graph associated to the digraph $G$. For example, we will denote by $U B(d, D)$ the undirected de Bruijn graph of maximum degree $2 d$, minimum degree $2 d-2(B(d, D)$ contains some loops) and diameter $D$. That is, the graph whose vertices are the words of length $D$ from an alphabet of $d$ letters in which the vertex $\left(x_{1}, \ldots, x_{D}\right)$ is joined to the vertices $\left(\alpha, x_{1}, \ldots, x_{D-1}\right)$ and $\left(x_{2}, \ldots, x_{D}, \alpha\right)$.

We will also denote by $U K(d, D)$ the undirected Kautz graph of maximum degree $2 d$, minimum degree $2 d-1(K(d, D)$ contains some parallel edges but no loop) and diameter $D$. That is, the graph whose vertices are the words of length $L$ from an alphabet of $d+1$ letters, with no two consecutive identical letters, in which the vertex $\left(x_{1}, \ldots, x_{D}\right)$ is joined to the vertices $\left(\alpha, x_{1}, \ldots, x_{D-1}\right)$ and $\left(x_{2}, \ldots, x_{D}, \alpha\right)$.

\section{Reliability properties in the directed case}

From the second definition (line digraph iterations), it can be shown that the connectivity of $K(d, D)$ is $d$ and that the connectivity of $B(d, D)$ is $d-1$. Indeed, the connectivity of $L(G)$ is at least the arc-connectivity of $G$. This was noted by different authors $([46,67,96,95])$. In $[67]$, M. Imase, T. Soneoka and K. Okada proved that 
$\Pi_{d, n}$ and $R P K_{d, n}$ are at least $(d-1)$-connected when their diameter is at least 4. That follows also from the result of J. Fabrega, M.A. Fiol and M. Escudero [45]
concerning $l(G)$.

Since $R P K_{d, n}$ has loops its connectivity is exactly $d-1$. Concerning the $\Pi_{d, n}$ graph, its connectivity has been determined recently by N. Homobono and $\mathrm{C}$. Peyrat [63]. It is not difficult to see that the connectivity of $\Pi_{d, n}$ is $d-1$ either when $d+1$ does not divide $n$ (in the case $\Pi_{d, n}$ has loops) or when $d$ and $n$ are relatively prime. Otherwise (that is, when $d+1$ divides $n$ and $d$ and $n$ are not relatively prime) N. Homobono and C. Peyrat [63] proved that the connectivity is $d$ when the diameter is at least 5 . The case when both $d$ and $d+1$ divide $n$ was proved independently by D.Z. Du and F.K. Hwang [38].

The diameter vulnerabilities of de Bruijn and Kautz digraphs have also been considered. In [68] M. Imase, T. Soneoka and K. Okada proved that in $B(d, D)$, there exist $d-1$ vertex disjoint paths, one of length at most $D$ and $d-2$ of length $D+1$. They also showed the existence of $d$ vertex disjoint paths between any pair of vertices in $K(d, D)$, one of length at most $D, d-3$ of length at most $D+1$ and two of length at most $D+2$. A quite similar result asserting the existence of $d-1$ paths of length at most $D+1$ and one of length at most $D+2$ is given in [95] but the proof seems to be incorrect. The above results show that the de Bruijn and Kautz digraphs are highly fault-tolerant. Indeed, if some nodes or arcs become faulty, the diameter of the resulting graph increases only by one or two, preserving the good properties of the network.

According to the properties of their surviving route graphs they are also very good networks. Indeed N. Homobono and C. Peyrat proved in [62] that $R(G, \rho) / F$ (where $G=B(d, D)$ or $G=K(d, D)$ ) has diameter 2, when $\rho$ is the unique minimal length routing and when $F$ is any set of faults of cardinality at most $\kappa(G)-1$. Generalizations of these results for iterated line digraphs have been obtained by M.A. Fiol, J. Fabrega, N. Homobono and M. Escudero [50]. In [79] M. Imase, Y. Manabe and T. Soneoka proved that $G=R P K_{d, n}$ has $\xi(G) \leq 2\left\lceil\log _{d} n\right\rceil$ and that furthermore, there exist a routing $\rho$ such that $\xi(G, \rho) \leq 3\left\lceil\log _{d} n\right\rceil$ and $D[R(G, \rho) / F] \leq$ 3 (if $n>d^{4}$ and $d>2$ ).

\section{Reliability results in the undirected case}

A.H. Esfahanian and S.L. Hakimi [43] proved that the de Bruijn undirected graph $U B(d, D)$ is maximally connected. Namely, the connectivity of $U B(d, D)$ is equal to
its minimum degree $2 d-2$.

A shorter proof of the connectivity of the de Bruijn undirected graph can be obtained in a similar way as that of the proof of Theorem A concerning Kautz
graphs, see [60].

Using results of his thesis [98], M.L. Schlumberger [99] had already proved that the connectivity of $U B(d, D)$ is $2 d-2$ when $D \geq 5$. In fact he proved that $U R P K_{d, n}$ is maximally connected if $n=p d^{k}$ where $p$ and $d$ are relatively prime and $k \geq 5$. Note that in [67] Appendix $A$ the authors also gave a theorem on the connectivity of $U R P K_{d, n}$, but the proof is incorrect. Recently N. Homobono [61] proved that $U R P K_{d, n}$ has connectivity $2 d-2$ if $D>4$ and $n>d^{D}$. 
We did rof find any result concerning the conncctivities of Kautz graphs in the literature, except the result in [67] whose proof is incorrect. In [6] J-C Bermond, N. Homobono and C. Peyrat showed that undirected Kautz graphs are also maximally connected.

Theorem A. The connectivity of the Kautz graph $U K(d, D)$ is $2 d-1$.

This result shows that the Kautz graphs are very suitable networks, better in fact than the de Bruijn graphs. For the same maximum degree and diameter, they have more vertices and a better connectivity (one less than the best possible one). We will see afterwards that one can construct a $2 d$-connected graph by adding some edges to $U K(d, D)$. In [61] N. Homobono proved that for $D>4$ and $n>d^{D}$, the connectivity of $U \Pi_{d, n}$ is $2 d-1$ if $d+1$ divides $n$ and $2 d-2$ otherwise.

Partial results concerning the diameter vulnerability of de Bruijn and Kautz graphs or some other families have been obtained by J. Bond and C. Peyrat [20] by considering these graphs as underlying graphs of line digraphs. For example they showed that the diameter of a graph obtained from $U B(d, D)$ or $U K(d, D)$ by deleting one vertex is still $D$, when $D$ and $d$ are not both equal to 2 . They also proved that after deletion of up to $2 d-2$ vertices, the diameter of $U K(d, D)$ increases at most by 2. A.H. Esfahanian and S.L. Hakimi [43] also showed that if at most $2 d-3$ nodes become faulty in $U B(d, D)$, there still exists a path of length at most $D+$ $\min \left(D, 5+\log _{d} D\right)$ between any two nodes.

Concerning the existence of vertex disjoint paths in $U B(d, D)$, the results by $\mathrm{M}$. Imase, T. Soneoka and K. Okada [68] implies the existence of $d-1$ vertex disjoint paths of length at most $D+1$. In [92] D.K. Pradhan and S.M. Reddy exhibited $d$ vertex disjoint paths of length at most $2 D$. In $[89,90]$ D.K. Pradhan proved that $R P K_{2, n}$ has diameter at most $3 \log _{2} n-1$ after deletion of any vertex.

The determination of the diameter of the surviving route graph associated to $U B(d, D)$ or to $U K(d, D)$ with a given routing is still an open problem. F.R.K. Chung, E.G. Coffman, M.I. Reiman and B.E. Simon [31] showed that undirected de Bruijn networks are asymptotically optimal in what concern the forwarding index, in that sense that they have the smallest possible forwarding index for a given number of vertices and given degree $\left(\xi(U B(d, D))\right.$ is of the order of $\left.\log _{d} n\right)$. N. Homobono [60] showed that Kautz networks are also asymptotically optimal. M-C Heydemann, J-C Meyer and D. Sotteau [58] proved that de Bruijn and Kautz networks are also asymptotically optimal for the edge forwarding index.

\section{Modified de Bruijn or Kautz graphs}

Since Kautz and de Bruijn graphs are not regular, some authors attempt to modify them in order to get regular, maximally connected graphs (that is, graphs of connectivity equal to the degree).

In [96] S.M. Reddy, D.K. Pradhan and J.G. Kuhl proposed to modify the de Bruijn digraph $B(d, D)$ in the following way: remove the $d$ self-loops and connect the vertices of degree $d-1$ into a particular cycle. They showed that the connectivity of this digraph is $d$. They also proposed another possible change: add a new 
vertex and connect it to all the vertices of degree $d-1$. The diameter is still $D$ and the connectivity is $d$.

V.P. Kumar and S.M. Reddy [74] obtained a $2 d$-regular graph from the Kautz undirected graph $U K(d, D)$ by adding a particular matching on the vertices of degree $2 d-1$, in such a way that the subgraph generated by these vertices is a cycle. They showed that the resulting graph has connectivity $2 d$. Furthermore, they gave a distributed and fault-tolerant routing which guarantees a path of length at most $D+4 t$ if $t<d$ nodes are faulty. They presented a routing strategy when $t<2 d$ nodes are faulty which results in a maximum path length of $3 D+6$ between any two non-faulty nodes.

In fact, J-C Bermond, N. Homobono and C. Peyrat showed that the graphs obtained from $U K(d, D)$ by adding any perfect matching between the vertices of degree $2 d-1$ have connectivity $2 d$, when $D \geq 4$ and $d \geq 3$.

Theorem B. For $d \geq 3$ and $D \geq 4$, any graph obtained from the undirected Kautz graph $U K(d, D)$ by adding a perfect matching on the vertices of degree $2 d-1$ has connectivity $2 d$.

\section{Construction of Large Reliable Networks}

Although Kautz graphs and their generalizations have nice reliability properties, it is interesting to construct other graphs having such properties. One problem for example consists in constructing maximally connected networks (graphs such that the connectivity is equal to the minimum degree) with minimum number of edges and minimum diameter. A particular case is to construct regular maximally connected networks. This problem has been considered by U. Schumacher [101] and S.M. Reddy, D.K. Pradhan and J.G. Kuhl [96]. A first attempt to solve the problem for digraphs has been done by A. Sengupta, A. Sen and S. Bandyodhyay [102] who have exhibited a family of $d$-regular digraphs of connectivity $d$ and diameter twice the lower bound. These digraphs have the additional property that the deletion of up to $d-2$ vertices increase the diameter by no more than 2 . Recently the problem has been settled by T. Soneoka, H. Nakada, M. Imase and Y. Manabe. In [105] they proved that the digraph obtained from $R P K_{d, n}$ by replacing the loops by a single circuit has maximum connectivity if $n>d^{3}$. In [103] they covered the remaining case.

A further step will consist in constructing graphs or digraphs having a hamiltonian cycle, or, if possible, many (the best possible being graphs decomposable into hamiltonian cycles). Indeed the routing on a loop is very simple. De Bruijn and Kaultz digraphs being line digraphs of eulerian digraphs are clearly hamiltonian. The results of Du, Hsu, Hwang and Zhang $[37,38]$ showed that $R P K_{d, n}$ is in fact hamiltonian except when $d=2$ and $n$ is odd and $\Pi_{d, n}$ is hamiltonian except if $d=2 n$ is odd and $n \neq 3^{k}$. When $d=2$ a class of networks called $H_{n}$ obtained by modifying the generalized Kautz digraphs has been proposed by Du, Hsu and Hwang [36]. They showed that $H_{n}$ is hamiltonian, has connectivity 2 and diameter at most $\left\lceil\log _{2}(n-1)\right\rceil+1$. 
Note that a graph which is decomposable into hamiltonian cycles has maximum possible edge-connectivity. Circulant graphs of degree 4, and, more generally, 4-regular connected Cayley graphs on abelian groups were recently shown to be always decomposable into two hamiltonian cycles [11]. However the general conjecture of $\mathrm{B}$. Alspach that any connected, $2 k$-regular Cayley graph on an abelian group can be decomposed into $k$ hamiltonian cycles remains open. More generally one can try to build graphs containing other interesting given subgraphs, like binary trees or grids or cubes. These embedding problem have been considered for the $D$-cubes (see the articles by S. Bhatt, F. Chung, T. Leighton and A. Rosenberg [13], D.S. Greenberg, L.S. Heath and A. Rosenberg [54], or A. Wagner [109]).

Another approach consists in constructing large $\left(\triangle, D, D^{\prime}, s\right)$-graphs, where a $\left(\Delta, D, D^{\prime}, s\right)$-graph is a $(\Delta, D)$-graph such that after the deletion of $s$ vertices the diameter is at most $D^{\prime}$. Constructions of such large graphs for $s=1$ and $D^{\prime} \in$ $\{D, D+1, D+2\}$ have been done by J. Bond and C. Peyrat [19]. Improvements of their results have been obtained by J. Gomez [53]. In [84] J. Opatrny, N. Srinivasan and V.S. Alagar give constructions of $(\Delta, D, D+1, \Delta-1)$-graphs for $D=3$ or 4 and $(\Delta, D, D+3, \Delta-1)$-graphs for $D=4$ or 5 , using Balanced Incomplete Block Designs. J. Opatrny [83] also constructed a family of 3-regular graphs with good fault tolerance. In [82], P. Morillo, M.A. Fiol and J. Guitart have constructed optimal $(d, 2,2, s)$-digraphs.

One can also construct large $(\triangle, D)$ graphs having a great number $p$ of vertex disjoint paths of length at most $D^{\prime}$. Such a problem with $p=\Delta$ (such a set of disjoint paths being called a "container") and $D^{\prime}=D+2$ was considered by F.J. Meyer and D.K. Pradhan [80] who constructed such graphs with $\Delta^{D / 2}$ vertices. Note that Kautz digraphs have this property with $p=d$ and $D^{\prime}=D+2$, as shown in [68]. The analogous problem for edge disjoint paths of length at most $D^{\prime}$ was asked by B. Elspas al. (see [94]) and partial results have been obtained by H.J. Quaife [94].

\section{References}

1. Akers, S.B., Harel, D., Krishnamurthy, B.: The star graph: an attractive alternative to the $n$-cube. 16th Conf. on Parallel Processing, 1987, pp. 393-400

2. Akers, S.B., Krishnamurthy, B.: On group graphs and their fault tolerance. IEEE Trans. Comput. C-36, 885-888 (1987)

3. Amar, D.: On the connectivity of some telecommunication networks. IEEE Trans. Comput. C-32, 512-519 (1983)

4. Ameter, D., de Gree, Max: Graphs and Interconnection networks. (Forthcoming book)

5. Berge, C.: Graphs and hypergraphs. New-York: North-Holland Publishing Co. (1973)

6. Bermond, J.-C., Homobono, N., Peyrat, C.: Large fault-tolerant interconnection networks. LRI Research Report, 1986

7. Bermond, J.-C., Bollobas, B.: The diameter of graphs- a survey. Proc. 12-th Southeastern Conference, Congressus Num. 32, 3-27 (1981)

8. Bermond, J.-C., Bond, J., Paoli, M., Peyrat, C.: Graphs and interconnection networks: diameter and vulnerability. In surveys in combinatorics: Proc. 9-th British Combinatorial Conference, London Math. Soc., Lec. Notes Ser. 82, 1-30 (1983)

9. Bermond, J.-C., Comellas, F., Hsu, F.: Distributed loop computer networks: a survey. IEEE trans. Comput. (1988) (submitted) 
10. Bermond, J.-C., Delorme, C., Quisquater, J.-J.: Strategies for interconnection networks: some methods from graph theory. J. of Parallel and Distributed Comput. 3, 433-449 (1986)

11. Bermond, J.-C., Favaron, O., Maheo, M.: Hamiltonian decomposition of Cayley graphs of degree four. J. Comb. Theory (B) (1988)

12. Bermond, J.-C., Illiades, G., Peyrat, C.: An optimization problem in distributed loop computer networks. In: Proc. third international conference on combinatorial math., New York, 1985, Annals N.Y. Acad. Science (1988)

13. Bhatt, S., Chung F.R.K., Leighton, T., Rosenberg, A.: Optimal simulations of tree machines. Proc. FOCS. pp. 274-282 (1986)

14. Boesch, F.T.: Graph theoretic models for network reliability studies. Stevens Institute of technology, electrical engineering and computer science department, Technical Report 8010, 1980

15. Boesch, F.T., Tindell, R.: Circulants and their connectivities. J. Graph Theory 8, 487-499 (1984)

16. Boesch, F.T., Wang, J.: Reliable circulant networks with minimum transmission delay. IEEE Trans. Circuits Syst. CAS-32, 1286-1291 (1985)

17. Bollobas, B.: Graphs with given diameter and minimal degree. Ars Comb. 2, 3-9 (1976)

18. Bond, J., Delorme, C., de la Vega, W.F.: Large Cayley graphs with small degree and diameter. LRI Research report 392, 1987

19. Bond, J., Peyrat, C.: Diameter vulnerability in networks. In: Proc. of Kalamazoo Colloquium 1984, Graph theory and its applications to algorithms and computer science. pp. 123-149, New York: Wiley 1985

20. Bond, J., Peyrat, C.: Diameter vulnerability of some large interconnection networks, The 19-th Southeastern Conference on Combinatorics, Graph Theory and Computing. Baton rouge, 1988

21. Bondy, J.A., Hell, P.: Counterexamples to theorems of Menger type for the diameter. Discrete Math. 44, 217-220 (1983)

22. Boyles, S.M., Exoo, G.: A counterexample to a conjecture on paths of bounded length. J. Graph Theory 6, 205-209 (1982)

23. Broder, A., Dolev, D., Fischer, M., Simons, B.: Efficient fault-tolerant routings in networks. In: Proc. ACM 16-th STOC. pp. 536-541. (1984)

24. de Bruijn, N.G.: A combinatorial problem. Koninklijke Nederlandse Academie van Wetenschappen Proc. A49, 758-764 (1946)

25. Campbell, L., Carlsson, G.E., Faber, V., Fellows, M.R., Langston, M.A., Moore, J.W., Mullhaupt, A.P., Sexton, H.B.: Dense symmetric networks from linear groups and codes (1988) (preprint)

26. Carlsson, G.E., Cruthirds, J.E., Sexton, H.B., Wright, C.G. Interconnection networks based on a generalization of cube-connected cycles. IEEE Trans. Comput. C-34, 769-772 (1985)

27. Chudnovsky, D.V., Chudnovsky, G.V., Denneau, M.M.: Regular graphs with small diameter as models for interconnection networks in 3rd Int. Conf. on Supercomputing Boston, 232-239 (1988)

28. Chung, F.R.K.: Diameter of communication networks. Proceedings of Symposia in Applied Mathematics 34, 1-18 (1986)

29. Chung, F.R.K.: Diameters of graphs: old problems and new results. In: Proc. 18-th southeastern conference on Combinatorics, Graph theory and Computing Congresses Numeration 60, 295-317 (1987)

30. Chung, F.R.K.: Graphs with small diameter after edge deletion (1988) (preprint)

31. Chung, F.R.K., Coffman, E.G., Reiman, M.I., Simon, B.E.: The forwarding index of communication networks. IEEE Trans. Informat. Theory. IT-33, 224-232 (1987)

32. Chung, F.R.K., Garey, M.R.: Diameter bounds for altered graphs. J. Graph Theory 8, $511-534(1984)$

33. Colbourn, C.J.: The Combinatorics of Networks Reliability. Oxford: Oxford University Press 1987

34. Dolev, D., Halpern, J., Simons, B., Strong, R.: A new look at fault-tolerant network routings. In: Proc. ACM, 16-th STOC. 526-535, 1984 
35. van Doorn, E.A.: Connectivity of circulant digraphs. J. Graph Theory 10, 9-14 (1986)

36. Du, D.Z., Hsu, D.F., Hwang, F.K.: Doubly-linked ring networks. IEEE Trans. Comput. C-34, $853-855(1985)$

37. Du, D.Z., Hsu, D.Z., Hwang, F.K., Zhang, X.M.: The hamiltonian property of generalized de Bruijn digraphs. J. Comb. Theory (to appear)

38. Du, D.Z., Hwang, F.K.: Generalized de Bruijn digraphs. Networks 18, $27-38$ (1988)

39. Dwork, C., Peleg, D., Pippenger, N., Upfal, E.: Fault tolerance in networks of bounded degree. SIAM J. Comput. (to appear)

40. Entringer, R.C., Jackson, D.E., Slater, P.J.: Geodetic connectivity of graphs. IEEE Trans. Circuits Syst. Cas-24, 460--463 (1977)

41. Escudero, M., Fabrega, J., Morillo, P.: Fault-tolerant routings in double-loop networks, In: Proc. 11-th British Combinatorical Conference 1987, Ars Comb. (to appear)

42. Esfahanian, A.H.: Lower-bounds on the connectivities of a graph. J. Graph Theory 9, 503-511 (1985)

43. Esfahanian, A.H., Hakimi, S.L.: Fault-tolerant routing in de Bruijn communication networks. IEEE Trans. Comput. C-34, 777-788 (1985)

44. Exoo, G.: On a measure of communication network vulnerability. Networks 12, 405-409 (1982)

45. Fábrega, J., Fiol, M.A., Escudero, M.: The connectivity of iterated line digraphs. Submitted to J. of Graph Theory

46. Fábrega, J., Fiol, M.A., Yebra, J.L.A., Alegre, I.: Connectivity and reliable routing algorithms in line digraphs. In: Proc. 3rd Int. Symp. Applied Informatics, pp. 45-50. Switzerland: Grindenwald 1985

47. Faudree, R.J., Ordman, E.T., Schelp, R.H., Jacobson, M.S., Tuza, Z.: Menger's theorem and short paths. J. Comp. Math. and Comp. Combinatorics 2, 235-253 (1987)

48. Favaron, O., Kouider, M., Maheo, M.: Edge-vulnerability and mean distance. Networks (to appear)

49. Feldman, P.: Fault tolerance of minimal path routings in a network. In: Proc. 17-th STOC, pp. 327-334, 1985

50. Fiol, M.A., Fábrega, J., Homobono, N., Escudero, M.: On surviving route graphs of iterated line digraphs. Submitted to Proc. of Kalamazoo Colloquium (1988)

51. Fiol, M.A., Yebra, A., Alegre, I., Valero, M.: A discrete optimization problem in local networks and data alignment. IEEE Trans. Comput. C-36, 702-713 (1987)

52. Fiol, M.A., Yebra, J.L.A., Alegre, I.: Line digraph iterations and the $(d, k)$ digraph problem. IEEE Trans. Computer. C-33, 400-403 (1984)

53. Gomez, J.: Diametro $y$ vulnerabilidad en redes de interconexion. Thesis, Universitat Politecnica de Catalunya, Barcelona, September 1986

54. Greenberg, D.S., Heath, L.S., Rosenberg, A.: Optimal embeddings of the FFT graph in the hypercube (1988) (preprint)

55. Hamidoune, Y.O.: On the connectivity of Cayley digraphs. Europ. J. Comb. 5, 309-312 (1984)

56. Hartman, J., Rubin, I.: On diameter stability of graphs. Lect. Notes Math. 642, 247-254 (1976)

57. Heydemann, M.C., Meyer, J.-C., Opatrny, J., Sotteau, D.: Consistent routings and forwarding indices (1988) (preprint)

58. Heydemann, M.C., Meyer, J.-C., Sotteau, D.: On forwarding indices of networks. Discrete App. Math. (1988)

59. Hillis, W.D.: The connection machine. In: ACM Distinguished Dissertation. The MIT press (1985)

60. Homobono, N.: Resistance aux pannes de grands reseaux d'interconnection. These de troisieme cycle, Universite de Paris-sud, LRI 1987

61. Homobono, N.: Connectivity of the undirected Imase and Itoh networks. In: Proc. 11-th British Conference, Ars Comb. 25C, 179-194 (1988)

62. Homobono, N., Peyrat, C.: Fault-tolerant routings in Kautz and de Bruijn digraphs. (To appear in the Combinatorial conference, Montreal, Quebec, 1987) 
63. Homobono, N., Peyrat, C.: Connectivity of Imase and Itoh digraphs. IEEE Trans. Comput. 37, 1459-1461 (1988)

64. Imase, M., Itoh, M.: Design to minimize a diameter on building block network. IEEE Trans. Comput. C-30, 439-443 (1981)

65. Imase, M., Itoh, M.: A design for directed graphs with minimum diameter. IEEE Trans. Comput. C-32, 782-784 (1983)

66. Imase, M., Manabe, Y.: Fault tolerant routing in a $\kappa$-connected network. Information Processing Letters, 1988

67. Imase, M. Soneoka, T., Okada, K.: Connectivity of regular directed graphs with small diameter. IEEE Trans. Comput. C-34, 267-273 (1985)

68. Imase, M., Soneoka, T., Okada, K.: A fault tolerant processor interconnection network. Denshi Tsushin Gakkai Ronbunshi J68-D, 8, 1449-1456 (in Japanese; translated in Systems and Computers in Japan, vol. 17, No. 8, 21-30, 1986.)

69. Itai, A., Perl, Y., Shiloach, Y.: The complexity of finding maximum disjoint paths with length constraints. Networks 12, 277-286 (1982)

70. Jolivet, J.-L.: Sur la connexite des graphes orientes. C.R. Acad. Sci., Paris, 274A, 148-150 (1972)

71. Kautz, W.H.: Bounds on directed $(d, k)$ graphs. Theory of cellular logic networks and machines, AFCRL-68-0668 Final Report, pp. 20-28, 1968

72. Kerjouan, R.: The edge-vulnerability of the diameter of interconnection networks. LRI Research Report 261, 1986, J. Graph Theory (submitted)

73. Krishnamoorthy, M.S., Krishnamurthy, B.: Fault diameter of interconnection networks. Comput. Math. Applic. 13, 577-582 (1987)

74. Kumar, V.P., Reddy, S.M.: A class of graphs for fault-tolerant processor interconnections. In: IEEE 1984 Int. Conf. Distributed Computing Systems, pp. 448-460, 1984

75. Lovász, L., Neumann-Lara, V., Plummer, M.: Mengerian theorems for paths of bounded length. Period. Math. Hung. 9, 269-276 (1978)

76. Mader, W.: Uber den Zusammenhang symmetrischer Graphen. Arch. Math. 21, 331-336 (1970)

77. Mader, W.: Minimale $n$-fach kantenzusammenhängende Graphen. Math. Ann. 191, 21-28 (1971)

78. Mader, W.: Connectivity and edge-connectivity in finite graphs. In: Surveys in Combinatorics: Proc. Seventh British Combinatorial Conference, Cambridge 1979, pp. 66-95. London Math. Soc. Lect. Note Ser. 38, (1979)

79. Manabe, Y., Imase, M., Soneoka, T.: Reliable and efficient fixed routings in digraphs. Invited paper, IECIE, Japan 1988

80. Meyer, F.J., Pradhan, D.K.: Flip-trees: fault-tolerant graphs with wide containers. IEEE Trans. Comput. 37, 472-478 (1988)

81. Morillo, P., Comellas, F., Fiol, M.A.: Diameter, mean distance and vulnerability of some graphs related to plane tesselations. Submitted to J. Graph Theory

82. Morillo, P., Fiol, M.A., Guitart, J.: On the $(d, D, D, s)$-digraph problem. 5th Int. Conf. on Applied Algebra, Error-Correcting Codes and Cryptography, AAECC-5, 1987

83. Opatrny, J.: A new family of 3-regular network models. In: Proc. of Int. Computer Symposium, Taiwan, pp. 1397-1401, 1986

84. Opatrny, J., Srinivasan, N., Alagar, V.S.: Highly fault-tolerant communication networks models. Submitted to IEEE on Circuits and System

85. Peleg, D., Simons, B.: On fault tolerant routings in general networks. PODCS, pp. 98-107, 1986

86. Peyrat, C.: Diameter vulnerability of graphs. Discrete Appl. Math. 9, 245-250 (1984)

87. Plesnik, J.: Note on diametrally critical graphs. In: Recent Advances in Graph Theory, Proc. 2-nd Czechoslovak Symp., Prague 1974, Academia Prague, pp. 455-465, 1975

88. Plesnik, J.: Critical graphs of given diameter Acta Fac. Rerum Natur. Univ. Com. Mat., 30, 71-93 (1975)

89. Pradhan, D.K.: Interconnecting topologies for fault-tolerant parallel and distributed architectures. In: Proc. ICPP, pp. 238-242, 1981 
90. Pradhan, D.K.: On a class of fault-tolerant multiprocessor network architectures. In: 3rd Int. Conf. on Distributed Processing, Miami, Floride, 1982

91. Pradhan, D.K.: Fault-tolerant Computing. Theory and Techniques, Englewood Cliffs, NJ, Prentice-Hall, 1986.

92. Pradhan, D.K., Reddy, S.M.: A Fault-tolerant communication architecture for distributed sustems. IEEE Trans. Comput. C-31, 863-870 (1982)

93. Preparata, F.R., Vuillemin, J.: The cube connected cycles: a versatile network for parallel computation. Commun. ACM 24, 300-309 (1981)

94. Quaife, H.J.: On $(d, k, \mu)$ graphs, IEEE Trans. Comput. C-18, 270-272 (1969)

95. Reddy, S.M., Kuhl, J.G., Hosseini, S.H., Lee, H.: On digraphs with minimum diameter and maximum connectivity. In: Proceedings of 20-th Annual Allerton Conference, pp. 1018-1026, 1982

96. Reddy, S.M., Pradhan, D.K., Kuhl, J.G.: Directed graphs with minimal diameter and maximum node connectivity. School of Engineering Oakland Univ., Tech. Rep., 1980

97. Ronen, D., Perl, Y.: Heuristics for finding a maximum number of disjoint bounded paths. Networks 14, 531-544 (1984)

98. Schlumberger, M.: De Bruijn communications networks. Standford PhD Thesis, Computer Science Department, Stanford, California, 1974

99. Schlumberger, M.: Connectivity of de Bruijn networks. Rapport de Recherche 154 Ensimag, Universite de Grenoble, 1978

100. Schoone, A.A., Bodlaender, H.L., van Leeuwen, J.: Diameter increase caused by edge deletion. J. Graph Theory 11, 409-427 (1987)

101. Schumacher, U.: An algorithm for construction of a $k$-connected graph with minimum number of edges and quasiminimal diameter. Networks 14, 63-74 (1984)

102. Sengupta, A., Sen, A., Bandyopadhyay, S.: On an optimally fault-tolerant multiprocessor network architecture. IEEE trans. Comp. C-36, 619-623 (1987)

103. Soneoka, T., Imase, M., Manabe, Y.: Design of a $d$-connected digraph with a minimum number of edges and a quasiminimal diameter, Part II. Submitted to Networks

104. Soneoka, T., Nakada, H., Imase, M.: Sufficient conditions for dense graphs to be maximally connected. In: Proc. of ISCAS 85, pp. 811-814, 1985

105. Soneoka, T., Nakada, H., Imase, M.: Design of a $d$-connected digraph with a minimum number of edges and quasiminimal diameter. Submitted to Discrete Appl. Math.

106. Soneoka, T., Nakada, H., Imase, M., Peyrat, C.: Sufficient conditions for maximally connected dense graphs. Discrete Math. 63, 53-66 (1987)

107. Suurballe, J.W.: Disjoint paths in a network. Networks 4, 125-145 (1974)

108. Vijayan, K., Murty, U.S.R.: On accessibility in graphs. Sankhya Ser. A, 26, 299-302 (1964)

109. Wagner, A.: Embedding arbitrary binary trees in a hypercube. Submitted to J. Parallel and Distributed Computing

110. Watkins, W.E.: Connectivity of transitive graphs. J. Comb. Theory 8, 23-29 (1970)

111. Wong, C.K., Coppersmith, D.: A combinatorial problem related to multimodule memory organizations. J. of Association for Communication for Computing Machinery 21, 392-402 (1974) 